\title{
TWO PROBABILITY THEOREMS AND THEIR APPLICATION TO SOME FIRST PASSAGE PROBLEMS
}

C. C. HEYDE

(received 23 July 1963)

\section{Introduction}

Let $X_{i}, i=1,2,3, \cdots$ be a sequence of independent and identically distributed random variables and write $S_{n}=X_{1}+X_{2}+\cdots+X_{n}$. If the mean of $X_{i}$ is finite and positive, we have $\operatorname{Pr}\left(S_{n} \leqq x\right) \rightarrow 0$ as $n \rightarrow \infty$ for all $x,-\infty<x<\infty$ using the weak law of large numbers. It is our purpose in this paper to study the rate of convergence of $\operatorname{Pr}\left(S_{n} \leqq x\right)$ to zero. Necessary and sufficient conditions are established for the convergence of the two series

$$
\sum_{n=1}^{\infty} n^{k} \operatorname{Pr}\left(S_{n} \leqq x\right), \quad-\infty<x<\infty
$$

where $k$ is a non-negative integer, and

$$
\sum_{n=1}^{\infty} e^{r n} \operatorname{Pr}\left(S_{n} \leqq x\right), \quad-\infty<x<\infty
$$

where $r>0$. These conditions are applied to some first passage problems for sums of random variables. The former is also used in correcting a queueing Theorem of Finch [4].

\section{Two Probability Theorems}

Let $X_{i}, i=1,2,3, \cdots$ be independent and identically distributed random variables. We write $S_{n}=X_{1}+X_{2}+\cdots+X_{n}, X_{i}^{-}=\min \left(0, X_{i}\right)$ and $X_{i}^{+}=X_{i}+X_{i}^{-}$.

We shall establish the following two Theorems:

THEOREM A ${ }^{1}$. Suppose $E|X|<\infty, E X>0$ and let $k$ be a non-negative

1 My attention has been drawn to a statement of Theorem A without proof in Smith, W. L. "On the elementary renewal theorem for non-identically distributed random variables" Univ. North Carolina Mimeographed Notes No. 352 (Feb. 1963). Professor Smith states that a proof of this result will appear in a paper entitled "On functions of characteristic functions and their applications to some renewal-theoretic random walk problems". 
integer. A necessary and sufficient condition for the convergence of the series

$$
\sum_{n=1}^{\infty} n^{k} \operatorname{Pr}\left(S_{n} \leqq x\right), \quad-\infty<x<\infty,
$$

is that $E|X-|^{k+2}<\infty$.

Theorem B. Suppose $E|X|<\infty$ and $E X>0$. A necessary and sufficient condition for the convergence of the series

$$
\sum_{n=1}^{\infty} e^{r n} \operatorname{Pr}\left(S_{n} \leqq x\right), \quad-\infty<x<\infty,
$$

for some $r>0$ is that $X^{-}$has an analytic characteristic function ${ }^{2}$.

(It is clear that analogous Theorems will hold in the case $E X<0$ ). We defer the proofs of Theorems A and B until some Lemmas have been established.

LEMMA 1. If $E|X|^{r}<\infty$ for some integer $r \geqq 1$ and $E X>0$, then

$$
\sum n^{r-2} \operatorname{Pr}\left(S_{n} \leqq x\right)<\infty, \quad-\infty<x<\infty .
$$

Proof: Write $E X=\mu$. Using Katz [5], Theorem 1, we have

$$
\sum n^{r-2} \operatorname{Pr}\left\{\left|S_{n}-n \mu\right| \geqq n \varepsilon\right\}<\infty \text {, every } \varepsilon>0
$$

from which we obtain, in particular

$$
\sum n^{r-2} \operatorname{Pr}\left(S_{n} \leqq(\mu-\varepsilon) n\right)<\infty \text {, every } \varepsilon>0 .
$$

Now we choose $\varepsilon$ so small that $\varepsilon<\mu$. We then have, for $n$ sufficiently large,

$$
\operatorname{Pr}\left(S_{n} \leqq x\right) \leqq \operatorname{Pr}\left(S_{n} \leqq(\mu-\varepsilon) n\right)
$$

and the result follows immediately from (1).

LEMMA 2. Let $E|X|<\infty$ and $E X>0$ or else $E|X|=\infty$ and, in either case, $E|X-|^{r}<\infty$ for some integer $r \geqq 1$. Then

$$
\sum n^{r-2} \operatorname{Pr}\left(S_{n} \leqq x\right)<\infty, \quad-\infty<x<\infty .
$$

Proof. We define a new random variable $Y$ as follows

$$
\begin{aligned}
Y & =X \text { if } X<K \\
& =0 \text { otherwise, }
\end{aligned}
$$

where tise crnstant $K(>0)$ is chosen so that $E Y>0$. Then, $Y \leqq X$ and $E|Y|^{r}<$, . It follows from Lemma 1 that

"The term "analytic characteristic function" is used for a characteristic function which is analytic in a strip containing the origin as an interior point. 


$$
\sum n^{r-2} \operatorname{Pr}\left(Y_{1}+Y_{2}+\cdots+Y_{n} \leqq x\right)<\infty, \quad-\infty<x<\infty .
$$

Also, if $X_{1}+X_{2}+\cdots+X_{n} \leqq x$, then $Y_{1}+Y_{2}+\cdots+Y_{n} \leqq x$ so that

$$
\operatorname{Pr}\left(Y_{1}+Y_{2}+\cdots+Y_{n} \leqq x\right) \geqq \operatorname{Pr}\left(X_{1}+X_{2}+\cdots+X_{n} \leqq x\right)=\operatorname{Pr}\left(S_{n} \leqq x\right)
$$

and hence

$$
\sum n^{r-2} \operatorname{Pr}\left(S_{n} \leqq x\right)<\infty, \quad-\infty<x<\infty .
$$

This completes the proof.

LEMMA 3. Let $E|X|<\infty, E X=\mu>0$ and

$$
\sum n^{k} \operatorname{Pr}\left(S_{n} \leqq x\right)<\infty, \quad-\infty<x<\infty
$$

for some non-negative integer $k$. Then $E \mid X^{-\left.\right|^{k+2}}<\infty$.

Our proof relies heavily on techniques used by Erdös [3].

Proof. Write $X_{i}^{*}=X_{i}-\mu$ and $Z_{n}=\sum_{i=1}^{n} X_{i}^{*}$. We then have

$$
\operatorname{Pr}\left(S_{n} \leqq x\right)=\operatorname{Pr}\left(Z_{n} \leqq x-n \mu\right) \geqq \operatorname{Pr}\left(Z_{n} \leqq-n c\right)
$$

for $c>\mu$ and $n$ sufficiently large.

Now from the fact that $E|X|<\infty$, it follows by a simple rearrangement that

$$
\sum_{n=1}^{\infty} \operatorname{Pr}\left(X_{i}^{*}<-(c+\varepsilon) n\right)<\infty,
$$

for arbitrary $\varepsilon>0$. Also, since the terms in this series are non-increasing, we have

$$
n \operatorname{Pr}\left(X_{i}^{*}<-(c+\varepsilon) n\right) \rightarrow 0 \text { as } n \rightarrow \infty .
$$

Write $\{E\}$ for the event $E$ and $\{E\}$ for the complement of $\{E\}$. We have

$$
\begin{array}{r}
\left\{Z_{n} \leqq-n c\right\} \supseteq \bigcup_{i=1}^{n}\left[\{ X _ { i } ^ { * } < - n ( c + \varepsilon ) \} \cap \left\{\mid X_{1}^{*}+\cdots+X_{i-1}^{*}+X_{i+1}^{*}+\cdots\right.\right. \\
\left.\left.+X_{n}^{*} \mid<(n-1) \varepsilon\right\}\right]
\end{array}
$$

and for the sake of brevity, we put

$$
\begin{aligned}
& A_{i}=\left\{X_{i}^{*}<-n(c+\varepsilon)\right\}, \\
& B_{i}=\left\{\left|X_{1}^{*}+\cdots+X_{i-1}^{*}+X_{i+1}^{*}+\cdots+X_{n}^{*}\right|<(n-1) \varepsilon\right\},
\end{aligned}
$$

$i=1,2,3, \cdots, n$. Thus, 


$$
\begin{aligned}
& \operatorname{Pr}\left(Z_{n} \leqq-n c\right) \geqq \operatorname{Pr}\left[\bigcup_{i=1}^{n}\left(A_{i} \cap B_{i}\right)\right] \\
& =\operatorname{Pr}\left[\bigcup_{i=1}^{n}\left\{\left(\overline{A_{1} \cap B_{1}}\right) \cap\left(\overline{A_{2} \cap B_{2}}\right) \cap \cdots \cap\left(\overline{A_{i-1} \cap B_{i-1}}\right) \cap\left(A_{i} \cap B_{i}\right)\right\}\right] \\
& =\sum_{i=1}^{n} \operatorname{Pr}\left[\left(\overline{A_{1} \cap B_{1}}\right) \cap \cdots \cap\left(\overline{A_{i-1} \cap B_{i-1}}\right) \cap\left(A_{i} \cap B_{i}\right)\right] \\
& \geqq \sum_{i=1}^{n} \operatorname{Pr}\left(\bar{A}_{1} \cap \bar{A}_{2} \cap \cdots \cap \bar{A}_{i-1} \cap A_{i} \cap B_{i}\right) \\
& \geqq \sum_{i=1}^{n}\left[\operatorname{Pr}\left(A_{i} \cap B_{i}\right)-\operatorname{Pr}\left\{\left(A_{1} \cup A_{2} \cup \cdots \cup A_{i-1}\right) \cap A_{i}\right\}\right] \\
& \geqq \sum_{i=1}^{n}\left[\operatorname{Pr}\left(B_{i}\right)-(i-1) \operatorname{Pr}\left(A_{1}\right)\right] \operatorname{Pr}\left(A_{i}\right) \\
& \geqq \sum_{i=1}^{n}\left[\operatorname{Pr}\left(B_{i}\right)-n \operatorname{Pr}\left(A_{1}\right)\right] \operatorname{Pr}\left(A_{i}\right) \text {. }
\end{aligned}
$$

Now take arbitrary $\rho, 0<\rho<1$ and $\delta>0$ such that $1-2 \delta \geqq \rho$. It follows from the weak law of large numbers that we can find an integer $N_{1}$ such that

$$
\operatorname{Pr}\left(B_{i}\right)>1-\delta \text { for } n \geqq N_{1} \text {. }
$$

Also, from (2), we can find an integer $N_{z}$-such that

$$
n \operatorname{Pr}\left(A_{1}\right)<\delta \text { for } n \geqq N_{2} \text {. }
$$

Thus, for $n \geqq \max \left(N_{1}, N_{2}\right)$, we have from (3)

$$
\operatorname{Pr}\left(Z_{n} \leqq-n c\right) \geqq n \rho \operatorname{Pr}\left(X_{i}^{*}<-(c+\varepsilon) n\right),
$$

and hence

$$
\sum n^{k+1} \operatorname{Pr}\left(X_{i}^{*}<-(c+\varepsilon) n\right)<\infty .
$$

We now introduce the random variable $Y$ defined by

and obtain

$$
\begin{aligned}
Y & =X^{*} \text { if } X^{*}<0 \\
& =0 \text { otherwise }
\end{aligned}
$$

$$
\sum n^{k+1} \operatorname{Pr}(Y<-(c+\varepsilon) n)<\infty .
$$

It follows from this, by a simple rearrangement, that $E|Y|^{k+2}<\infty$ and hence that $E \mid X^{-\left.\right|^{k+2}}<\infty$. This completes the proof.

Proof of Theorem A. Theorem A follows immediately from Lemmas 2 and 3 .

We now go on to give two Lemmas leading up to a proof of Theorem B. 
The development of the proof is similar to that used above in the proof of Theorem A.

Lemma 4. Suppose $X^{-}$has an analytic characteristic function and either $E|X|<\infty$ and $E X>0$ or $E|X|=\infty$. There exists a constant $R>0$ such that

$$
\sum e^{r n} \operatorname{Pr}\left(S_{n} \leqq x\right)<\infty
$$

for every $x,-\infty<x<\infty$ and every $r, 0<r<R$.

Proof. Since $X^{-}$has an analytic characteristic function, there exists a constant $K>0$ such that

$$
\Phi(\theta)=E\left(e^{-\theta \boldsymbol{x}}\right)<\infty
$$

for all $\theta$ in $0 \leqq \theta<K$. Now for such a $\theta$, a well-known Chebyshev type inequality gives

$$
\operatorname{Pr}\left(S_{n} \leqq x\right) \leqq e^{\theta x} E\left(e^{-\theta S_{n}}\right)=e^{\theta x}\{\Phi(\theta)\}^{n} .
$$

Also, in view of our assumption that $E\left|X^{-}\right|<\infty$ and either $E|X|<\infty$ and $E X>0$ or $E|X|=\infty$, we must have $\Phi(\theta)<1$ for sufficiently small positive $\theta$. We then choose $R$ so small that $e^{R} \Phi(\theta)<1$ and for all $r$, $0<r<R$,

$$
\sum e^{r n} \operatorname{Pr}\left(S_{n} \leqq x\right)<\infty .
$$

This completes the proof of the Lemma. I am indebted to the referee for this direct proof. My original proof was based on Baum, Katz and Read [1], Theorem 2, 190.

Lemma 4 is a generalization of the well-known result of Stein [9] which deals with the case $X^{-}=0$. It should be noted that although Stein's result is correct, his proof is invalidated by a misinterpretation of the Markov chain property of the sequence $\left\{S_{n}\right\}$ of sums.

Lemma 5. Let $E|X|<\infty$. Suppose $E X=\mu>0$ and

$$
\sum e^{+n} \operatorname{Pr}\left(S_{n} \leqq x\right)<\infty
$$

for all $r, 0<r<R$ and all $x,-\infty<x<\infty$. Then $X^{-}$has an analytic characteristic function.

PROof. We retain the notation of Lemma 3. Proceeding precisely as in Lemma 3, we obtain (4)

$$
\operatorname{Pr}\left(Z_{n} \leqq-n c\right) \geqq n \rho \operatorname{Pr}\left(X_{i}^{*}<-(c+\varepsilon) n\right),
$$

so that certainly

$$
\sum e^{r n} \operatorname{Pr}\left(X_{i}^{*}<-(c+\varepsilon) n\right)<\infty .
$$


We now introduce the random variable $Y$ defined by

and obtain

$$
\begin{aligned}
Y & =X^{*} \text { if } X^{*}<0 \\
& =0 \text { otherwise, }
\end{aligned}
$$

$$
\sum e^{r n} \operatorname{Pr}(Y<-(c+\varepsilon) n)<\infty .
$$

It follows immediately, using Lukacs [7], Theorem 7.2.1, 137, that $Y$ and hence $X^{-}$has an analytic characteristic function. This completes the proof of the Lemma.

Proof of Theorem B: Theorem B follows immediately from Lemmas 4 and 5.

It is worth remarking that it is quite likely that in Theorems $A$ and B the condition $E|X|<\infty, E X>0$ can be replaced by the condition $E|X|<\infty, E X>0$ or $E|X-1<\infty, E| X \mid=\infty$.

\section{Application to some first passage problems}

Let $X_{i}, i=1,2,3, \cdots$ be independent and identically distributed random variables and write $S_{n}=X_{1}+X_{2}+\cdots+X_{n}$. Consider a single boundary at $A(\geqq 0)$ so that if

$$
\begin{aligned}
& F_{0}(x)= \begin{cases}1 & x \geqq 0 \\
0 & x<0,\end{cases} \\
& F_{1}(x)=\operatorname{Pr}\left(S_{1} \leqq x\right), \\
& F_{n}(x)=\operatorname{Pr}\left(S_{n} \leqq x ; \max _{1 \leqq k \leqq n-1} S_{k} \leqq A\right), n>1,
\end{aligned}
$$

the probability $p_{n}$ that the first passage time out of the interval $(-\infty, A]$ for the process $S_{n}$ is $n$ is given by

$$
p_{n}=F_{n-1}(A)-F_{n}(A), \quad n \geqq 1 .
$$

This passage problem in the case $A=0$ arises, for example, in the busy period distribution of the queue $G I / G / 1$ which has been considered by various authors such as Finch [4].

We introduce the probability generating function $P(\lambda)=\sum_{r-1}^{\infty} \lambda^{r} p_{r}$ for the first passage time distribution (henceforth abbreviated F.P.T.D.) $\operatorname{Pr}(N=n)=p_{n}$. We have formally

$$
\begin{aligned}
& P^{\prime}(1)=E(N)=1+\sum_{r=1}^{\infty} F_{r}(A) \\
& P^{\prime \prime}(1)=\sum_{r=2}^{\infty} r(r-1) p_{r}=E\left(N^{2}\right)-E(N)=2 \sum_{r=1}^{\infty} r F_{r}(A),
\end{aligned}
$$


and in general for $k>1$,

$$
\begin{aligned}
P^{(k)}(1) & =(\alpha)_{k}(\text { the } k \text {-th factorial moment of } N) \\
& =k \sum_{r=k-1}^{\infty}(r)_{k} F_{r}(A)=\sum_{r=0}^{k} s(k, r) E\left(N^{r}\right)
\end{aligned}
$$

where $(r)_{k}=r(r-1)(r-2) \cdots(r-k+1)$ and $s(k, r)$ are the Stirling numbers of the first kind. It is thus clear that $E\left(N^{r}\right)<\infty$ for some positive integer $r$ if and only if $\sum n^{r-1} F_{n}(A)<\infty$. Also, the random variable $N$ has an analytic characteristic function if and only if the radius of convergence of $P(\lambda)$ is greater than unity or equivalently if $\sum e^{r n} F_{n}(A)<\infty$ for some $r>0$.

Now we write

$$
q_{n}=\operatorname{Pr}\left(\max _{1 \leqq k \leqq n} S_{k} \leqq 0\right), \quad n \geqq 1 ; \quad q_{0}=1 .
$$

Spitzer [8], 332, shows that

$$
\sum_{n=0}^{\infty} q_{n} t^{n}=\exp \left\{\sum_{n=1}^{\infty} \frac{t^{n}}{n} \operatorname{Pr}\left(S_{n} \leqq 0\right)\right\}
$$

a result originally due, in a slightly different form, to E. Sparre Andersen. From this we obtain

$$
q_{n} \geqq \frac{1}{n} \operatorname{Pr}\left(S_{n} \leqq 0\right)
$$

Thus,

$$
\operatorname{Pr}\left(S_{n} \leqq A\right) \geqq \operatorname{Pr}\left(\max _{1 \leqq k \leqq n} S_{k} \leqq A\right)=F_{n}(A) \geqq q_{n} \geqq \frac{1}{n} \operatorname{Pr}\left(S_{n} \leqq 0\right),
$$

and we readily obtain from Theorem $B$ :

THEOREM 1. The F.P.T.D. generated by the random variable $X$ with $E|X|<\infty$ and $E X>0$ has an analytic characteristic function if and only if $X^{-}$has an analytic characteristic function.

Further, we obtain immediately from Theorem A:

THEOREM 2. Let $r>1$ be a positive integer. Consider the F.P.T.D. generated by the random variable $X$ with $E|X|<\infty$ and $E X>0$. If the F.P.T.D. has a finite $r$-th moment, then $E\left|X^{-}\right| r<\infty$. If, on the other hand, $E|X-|^{r}<\infty$, then the F.P.T.D. has finite moments at least $u p$ to the $(r-1)$ th.

In the particular case where $A=0$, we can improve this Theorem by virtue of the relation (6). In fact, formally differentiating (6) $(r-1)$ times, we see that

$$
\sum n^{r-2} \operatorname{Pr}\left(S_{n} \leqq 0\right)<\infty \text { if and only if } \sum n^{r-1} q_{n}<\infty .
$$


Thus, in view of our comments above, the $r$-th moment of the F.P.T.D. exists if and only if

$$
\sum n^{r-2} \operatorname{Pr}\left(S_{n} \leqq 0\right)<\infty
$$

We therefore obtain immediately from Theorem A:

TheOREM 3. Let $r>1$ be a positive integer. The zero-barrier F.P.T.D. generated by the random variable $X$ with $E|X|<\infty$ and $E X>0$ has a finite $r$-th moment if and only if $X^{-}$has a finite $r$-th moment.

Before ending this section, it is worth remarking that Derman and Robbins [2] show that it is possible to have $E\left|X^{+}\right|=\infty, E\left|X^{-}\right|=\infty$ and $\operatorname{Pr}\left(S_{n}>0\right.$ i.o. $)=1, \operatorname{Pr}\left(S_{n} \leqq 0\right.$ i.o. $)=0$ and hence, following Kemperman [6], Theorem 15.2, 81, $\sum 1 / n \operatorname{Pr}\left(S_{n}>0\right)=\infty, \sum 1 / n \operatorname{Pr}\left(S_{n} \leqq 0\right)<\infty$. This provides us with a limitation on eventual improvements of the Theorems given above.

\section{Correction to a theorem of Finch [4]}

Let $\eta$ be the difference between the inter-arrival and service time in a $G I / G / 1$ queue. We refrain from stating the usual queueing assumptions for the sake of brevity. Let $\Pi_{n}$ be the probability that $n$ customers are served in a busy period. Then, as is well known,

$$
\begin{aligned}
& \Pi_{1}=\operatorname{Pr}\left(\eta_{1}>0\right) \\
& \Pi_{n}=\operatorname{Pr}\left(\max _{1 \leqq k \leq n-1} \eta_{1}+\eta_{2}+\cdots+\eta_{k} \leqq 0, \eta_{n}>0\right), \quad n>1,
\end{aligned}
$$

so that $\operatorname{Pr}(T=n)=\Pi_{n}$ is a zero-barrier F.P.T.D.

Finch [4] gives the following Theorem (his Theorem 2, 223).

Theorem Suppose that $E|\eta|<\infty$. Write $\Pi=\sum_{j=1}^{\infty} \Pi_{j}, N=\sum_{j=1}^{\infty} j \Pi_{j}$, and $a_{n}=\operatorname{Pr}\left(\eta_{1}+\eta_{2}+\cdots+\eta_{n}>0\right)$. Then,

$$
\begin{aligned}
& I= \begin{cases}1 & \text { if } E \eta \geqq 0 \\
1-\exp \left\{-\sum_{n=1}^{\infty} n^{-1} a_{n}\right\} & \text { if } E \eta<0\end{cases} \\
& N= \begin{cases}\exp \left\{\sum_{n=1}^{\infty} n^{-1}\left(1-a_{n}\right)\right\} & \text { if } E \eta>0 \\
\infty & \text { if } E \eta=0 \\
\sum_{n=1}^{\infty} a_{n} \exp \left\{-\sum_{n=1}^{\infty} n^{-1} a_{n}\right\} & \text { if } E \eta<0 .\end{cases}
\end{aligned}
$$

It is the final part of the statement of this Theorem that is incorrect, namely that 


$$
N=\sum_{n=1}^{\infty} a_{n} \exp \left\{-\sum_{n=1}^{\infty} n^{-1} a_{n}\right\}<\infty \text { if } E \eta<0 .
$$

In fact, under the condition $E_{\eta}<0$, we see from a negative mean analogue of Theorem $A$ that $\sum_{n=1}^{\infty} a_{n}<\infty$ if and only if $E\left|\eta^{+}\right|^{2}<\infty$. Thus, $N=\infty$ if $E \eta<0$ and $E\left|\eta^{+}\right|^{2}<\infty$. Finch's error arises from an invalid application of the Borel zero-one criterion which yields $\operatorname{Pr}\left(S_{n}>0\right.$ i.o. $)=0$ or 1 according as $\sum a_{n}<\infty$ or $=\infty$. Actually, using Kemperman [6], Theorem $15.2,81, \operatorname{Pr}\left(S_{n}>0\right.$ i.o. $)=0$ or 1 according as $\sum n^{-1} a_{n}<\infty$ or $=\infty$. Finch's Theorem and his proof of it can easily be repaired in terms of these comments. A correct statement of the Theorem is as follows:

THEOREM. Suppose that $E|\eta|<\infty$. Write $\Pi=\sum_{j=1}^{\infty} \Pi_{f}, N=\sum_{j=1}^{\infty} j \Pi_{f}$, and $a_{n}=\operatorname{Pr}\left(\eta_{1}+\eta_{2}+\cdots+\eta_{n}\right)>0$. Then

$$
\begin{aligned}
& I= \begin{cases}1 & \text { if } E \eta \geqq 0 \\
1-\exp \left\{-\sum_{n=1}^{\infty} n^{-1} a_{n}\right\} & \text { if } E \eta<0\end{cases} \\
& N= \begin{cases}\exp \left\{\sum_{n=1}^{\infty} n^{-1}\left(1-a_{n}\right)\right\} & \text { if } E \eta>0 \\
\infty & \text { if } E \eta=0 \text { or } E \eta<0 \text { and } E\left|\eta^{+}\right|^{2}=\infty \\
\sum_{n=1}^{\infty} a_{n} \exp \left\{-\sum_{n=1}^{\infty} n^{-1} a_{n}\right\} & \text { if } E \eta<0 \text { and } E\left|\eta^{+}\right|^{2}<\infty .\end{cases}
\end{aligned}
$$

\section{References}

[1] Baum, L. E., Katz, M. L., and Read, R. R., Exponential convergence rates for the law of large numbers, Trans. Amer. Math. Soc., 102 (1962), $187-199$.

[2] Derman, C., and Robbins, H., The strong law of large numbers when the first moment does not exist, Proc. Nat. Acad. Sci. U.S.A., 41 (1955), 586-587.

[3] Erdös, P., On a theorem of Hsu and Robbins, Ann. Math. Statist., 20 (1949), 286-291.

[4] Finch, P. D., On the busy period in the queueing system $G I / G / 1, J$. Austral. Math. Soc., 2 (1961), 217-228.

[5] Katz, M. L., The probability in the tail of a distribution, Ann, Math. Statist., 34 (1963), $312-318$.

[6] Kemperman, J. H. B., The passage problem for a stationary Markov chain, Univ. of Chicago Press (1961).

[7] Lukacs, E., Characteristic functions, Griffin, London (1960).

[9, Spitzer, F., A combinatorial lemma and its applications to probability theory, Trans. Amer. Malh. Soc., 82 (1956), 323-339.

[9] Stein, C., A note on cumulative sums, Ann. Math. Statist., 17 (1946), 498-499.

The Australian National University, Canberra 